\title{
Imagem no traumatismo abdominal
}

\author{
Imaging in abdominal trauma
}

\author{
Shri Krishna Jayanthi
}

Jayanthi SK. Imagem no traumatismo abdominal/Imaging in abdominal trauma. Rev Med (São Paulo). 2011 out.-dez.;90(4):195-200.

RESUMO: O trauma ocorre em eventos cinéticos com transmissão de energia e danos em graus variáveis em diversos órgãos. Afetando pacientes jovens resulta em grande impacto social. $O$ atendimento a este paciente deve ser o mais eficiente possível para reduzir esse impacto. Neste contexto específico, o trauma abdominal é um evento frequente. A avaliação clínica abdominal muitas vezes é limitada diante do potencial de lesões ocultas. Os métodos de imagem auxiliam nesta investigação delineando as lesões. A radiografia convencional apresenta papel menor, cabendo os principais papéis à ultrassonografia, realizada com protocolo de pesquisa de líquido livre como método inicial de triagem, e à tomografia computadorizada, o atual método de referência na busca direta das lesões intra-abdominais. Desta maneira, armado com os dados clínicos, os dados subsidiários dos exames de imagem permitem a conduta adequada para cada caso.

DESCRITORES: Traumatismos abdominaias/radiografia; Traumatismos abdominais/ultrassonografia; Ferimentos e lesões.
Abstract: Trauma occurs in events with kinetic energy transmission and damage in varying degrees to different organs. Affecting young patients it results in major social impact. The care of these patients should be carried out in the most efficient way to reduce this impact. In the specific context, abdominal trauma is a frequent event. Clinical evaluation is often limited due to potential hidden injuries. Diagnostic imaging assist this investigation outlining these lesions. Conventional radiograph shows minor role, with the main roles remaining for ultrasound, performed in protocol searching for free fluid as the initial screening method, and computed tomography, the current reference method searching for intraabdominal injuries. Thus, associating clinical data and subsidiary imaging data, the surgeon is allowed to choose the appropriate action for each case.

KEYWORDS: Abdominal injuries/radiography; Abdominal injuries/ ultrasonography; Wounds and injuries.

Médico chefe de setor de Radiologia de Emergência do Hospital das Clínicas da Faculdade de Medicina da Universidade de São Paulo.

Endereço para correspondência: InRad-HCFMUSP. Av. Dr. Enéas de Carvalho Aguiar, 255 - Cerqueira César, São Paulo, SP. CEP: 05403-001. E-mail: skjyanthi@yahoo.com.br 


\section{INTRODUÇÃO}

$\mathrm{O}$ traumatismo ocorre em eventos cinéticos com transferência e dissipação de energia pelo contato entre os corpos envolvidos no choque, resultando em lesões em diversas localidades e em múltiplos órgãos e estruturas. Nos séculos $X X$ e neste, o automóvel (cada vez mais rápido e popular) e as edificações (cada vez mais altas e disseminadas) são os principais vilões, pois fornecem a energia necessária para gerar o dano.

O traumatismo é a principal causa de morbidade e mortalidade no grupo que engloba da criança ao adulto, desde os cinco aos quarenta anos, com proporção aproximadamente de quatro homens: uma mulher. Considerando a faixa etária temos um grupo economicamente ativo, assim o impacto social e econômico do politraumatismo é grande. No Brasil, o impacto estimado das mortes por causa externa, incluindo a violência é de aproximadamente 20 bilhões de reais, calculada para o ano de 2001.

Para reduzir o impacto, as ações necessárias são amplas e envolvem esforços em diversas frentes: sanitária, com a redução do consumo de álcool; infraestrutura, com melhoria das vias de tráfego e aumento de segurança para pedestres; industrial ao se desenvolver novos equipamentos e metodologias de segurança; legais, utilizando-se regras mais rígidas com sua efetiva vigência e fiscalização; educação específica ao trânsito; e social, ao se reduzir índices de pobreza e desigualdade, com o intuito de ser reduzir a violência.

No campo médico, a preocupação sobre o tema iniciou-se durante a década de 60 e apenas na década de 80 foi criada uma padronização no atendimento inicial ao politraumatizado com a instituição do ATLS. O objetivo era evitar condutas divergentes e heterogêneas, com a atenção à vítima se iniciando desde o local do acidente. Incluemse neste atendimento equipes multiprofissionais, principalmente as paramédicas e nota-se o natural surgimento da avaliação e conduta por sistemas orgânicos e prioridades específicas.

Os recursos de diagnóstico por imagem sempre prestaram apoio ao diagnóstico e decisão nesse contexto. O progressivo desenvolvimento tecnológico permitiu novas modalidades com aumento dos recursos para o diagnóstico de potenciais lesões.

$\mathrm{O}$ sincronismo entre as equipes radiológica e cirúrgica é fundamental para que haja um adequado fluxo padronizado quanto à sequência de exames e a interpretação dos mesmos.

Dentro do complexo do politraumatismo, o traumatismo abdominal fechado é um evento bastante frequente e com certa dificuldade quanto à sua avaliação e manejo. Sua particularidade reside na possibilidade de lesões em graus variáveis em diferentes estruturas intra-abdominais, sem a presença de lesões externas ou com poucos sintomas abdominais.

\section{DIAGNÓSTICO}

A detecção do hemoperitônio foi considerada como um dos métodos de avaliação de possíveis lesões traumáticas intra-abdominais sendo que um dos primeiros métodos preconizados para sua detecção foi a punção abdominal diagnóstica, que apresenta baixa sensibilidade, mesmo utilizandose a técnica de quatro quadrantes. Posteriormente a técnica foi modificada, com a introdução da lavagem peritoneal diagnóstica em 1965, com grande impacto na detecção de suspeita de lesões intraabdominais.

A lavagem peritoneal consiste num procedimento invasivo, que envolve um pequeno procedimento cirúrgico, com a introdução de uma sonda na cavidade peritoneal com infusão de soro fisiológico, posteriormente recuperado e analisado. A positividade do exame é determinada pela presença de sangue neste material recuperado que somado aos demais dados clínicos e laboratoriais resulta na conduta a ser tomada. Ainda persiste como principal método de avaliação de hemoperitônio em alguns centros que não contam com equipamentos de diagnóstico por imagem que não a radiografia convencional. Tal procedimento, apesar da alta sensibilidade, envolve alguns inconvenientes, sendo os principais: consistir um procedimento cirúrgico com seus riscos, não conseguir quantificar o hemoperitônio, não permitir a determinação do órgão-alvo e o grau da lesão, que muitas vezes é autolimitada, além de reveses menores e infrequentes como resultados dúbios e não aplicabilidade em alguns casos, levando a inferências erradas com consequente impacto sobre as condutas.

O diagnóstico por imagem sempre se prestou a auxiliar na avaliação do traumatismo, sendo a radiologia convencional a primeira modalidade disponível. Esta permite uma avaliação inicial abdominal bastante limitada, porém sensibilizando a suspeita de lesões através da detecção de pneumoperitônio (achado pouco frequente), de significativas quantidades de líquido, de alterações na distribuição de alças e da concomitância de fraturas (costelas, bacia e coluna). Ressalve-se que esta última particularidade ainda é bastante útil atualmente (Figuras 1 e 2). 


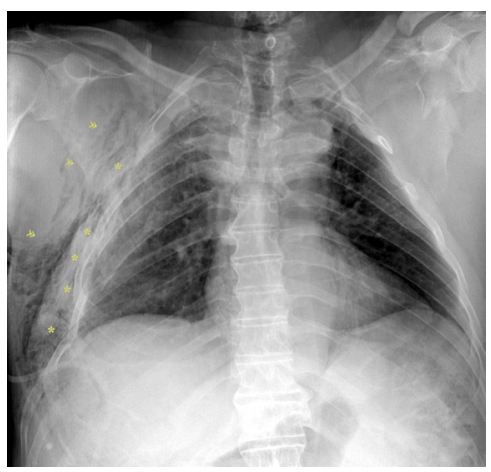

Figura 1. RX de Tórax: Fraturas de costelas à direita ( $\left.{ }^{*}\right)$. Enfisema de subcutâneo associado (»)

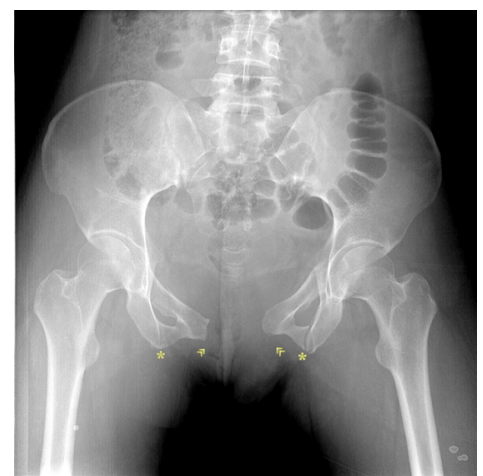

Figura 2. RX de Bacia: Disjunção da sínfise púbica, fratura em "livro aberto" (») e fratura dos ramos isquiopúbicos $\left(^{*}\right)$

Posteriormente, com o desenvolvimento de meios de contraste radiológicos, a radiologia contrastada permitiu a avaliação, ainda que parcial e algo limitada, de lesões específicas, particularmente: segmentos de vísceras ocas (estudos contrastados gastrointestinais), estruturas vasculares e órgãos parenquimatosos como fígado, rins e baço (radiologia vascular e urografia excretora) e para a avaliação do trauma uretral (Figura 3); possivelmente esta e a radiologia vascular ainda são as únicas indicações formais da modalidade e eventual tratamento, no caso da radiologia vascular. Não são superiores à lavagem peritoneal diagnóstica quanto à detecção do hemoperitônio, mas permitem a avaliação específica de lesões em órgãos-alvo.

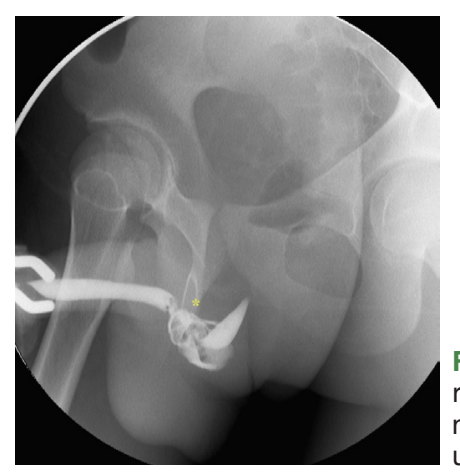

Figura 3. Uretrocistografia retrógrada: Lesão traumática no segmento bulbar da uretra $(*)$
Com o desenvolvimento tecnológico e disseminação de equipamentos, os meios de diagnóstico por imagem passaram a exercer importante papel nesta avaliação, principalmente através da ultrassonografia e da tomografia computadorizada.

A ultrassonografia foi primeiramente descrita como método alternativo para a detecção de lesões intra-abdominais traumáticas na década de 70 . Posteriormente outros trabalhos foram demonstrando mais vantagens da ultrassonografia, estabelecendose como método alternativo à lavagem peritoneal diagnóstica na detecção de hemoperitônio a partir da década de 90.

Atualmente a ultrassonografia é efetivamente utilizada como método inicial para detecção de líquido livre intraperitoneal, recebendo o acrônimo de FAST - Focused Assessment with Sonography for Trauma (Avaliação Ultrassonográfica Focalizada no Trauma). O exame é realizado na própria sala de emergência, ainda durante o atendimento inicial, junto com a exposição e avaliação global. A dinâmica deste exame nesse contexto envolve grande interação com o restante da equipe de atendimento, com o resultado imediatamente comunicado, permitindo a tomada das decisões de maneira rápida.

A sensibilidade para a detecção de líquido livre intraperitoneal é alta, atingindo $100 \%$ para 50 $\mathrm{mL}$, sendo esse o principal objetivo do exame. Para a detecção de lesões de órgãos parenquimatosos a sensibilidade ainda é baixa. Assim, o desempenho da ultrassonografia na detecção de líquido livre é superior à detecção de lesão parenquimatosa.

Como outras desvantagens, há a limitação na realização do exame em alguns contextos como obesidade, enfisema de subcutâneo e lesões de pele que impeçam o contato do transdutor com o paciente. Depende também de um examinador com experiência adequada para a correta interpretação dos resultados, uma vez que algumas armadilhas existem para gerar resultados dúbios.

A tomografia computadorizada (TC) é considerada atualmente como padrão-ouro para avaliação do traumatismo abdominal, pois permite uma ampla avaliação abdominal, detectando desde pequenas quantidades de líquido livre, inferindo hemoperitônio, bem como minuciosa avaliação de estruturas parenquimatosas e vísceras ocas, mesentério, retroperitônio e parede abdominal. Com o advento da TC, muda-se o paradigma na detecção de lesões: ao invés de se preocupar na detecção do hemoperitônio, passa-se a procurar diretamente a lesão. Muitas vezes é o principal exame na determinação da conduta, se expectante ou não. Apresenta como principais fatores limitantes: o uso de equipamento ainda não 
tão difundido e que apresenta investimento inicial e de manutenção altos, resultando num exame com um custo relativamente elevado; a necessidade de transporte do paciente até o equipamento, que pode ser inconveniente em alguns casos em virtude das condições clínicas; o uso de contraste endovenoso, que têm potencial alergênico e nefrotóxico e a exposição à radiação ionizante.

Considerando-se o risco/benefício num contexto de trauma abdominal fechado com potencial lesão intra-abdominal, esses últimos dois fatores e o deslocamento do paciente, passam a ser riscos menores, onde o benefício do exame compensaria os riscos expostos. Ainda assim, persiste a questão do custo do exame, que individualmente seria relativamente baixo, porém considerando-se o movimento de um serviço de emergências com um grande número de casos de politraumatismo, tal custo poderia tornar-se proibitivo.

\section{REALIZAÇÃO DOS EXAMES}

A ultrassonografia com protocolo FAST baseiase na pesquisa rápida de líquido livre. O exame é realizado com o equipamento à beira do leito, realizando pesquisa na seguinte ordem: janela pericárdica, hipocôndrio direito, hipocôndrio esquerdo, aorta e pelve (Figura 4).

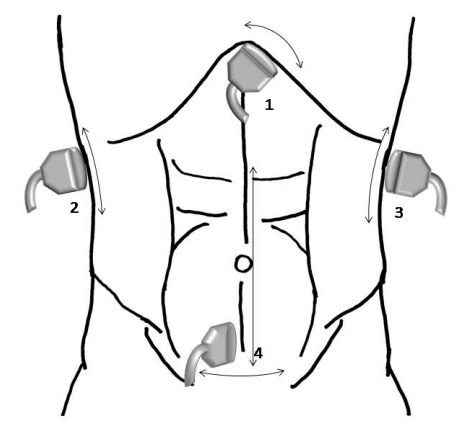

Figura 4.Protocolo de realização do US FAST compreendendo janela pericárdica (1), hipocôndrio direito (2), hipocôndrio esquerdo (3) e aorta e pelve (4)

O exame positivo caracteriza-se pela presença de líquido livre em qualquer quantidade em qualquer uma das janelas em que se acessa o exame (Figura 5). Ressalve-se que em mulheres em idade fértil a presença de pequenas quantidades de líquido livre seja comum, sem necessariamente indicar doença. Porém este dado deve ser olhado com bastante cautela e outros quadrantes têm que ser investigados para que não se desvalorize desnecessariamente o único achado positivo que pode indicar eventual lesão.
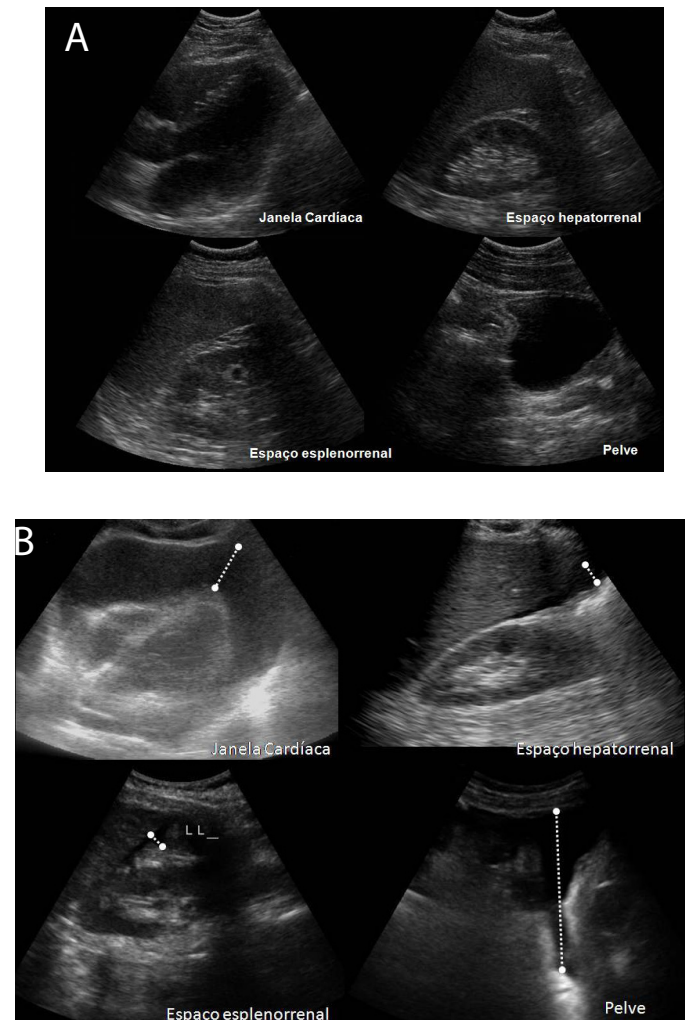

Figura 5. Pesquisa de líquido livre no US FAST: exame negativo - normal (a) e exame positivo (b)

O local mais comum de se achar líquido livre em caso de presença de lesão é o hipocôndrio direito, seguido pela pelve e pelo hipocôndrio esquerdo. Líquido livre interalças também pode ser detectado, porém isso pode indicar uma maior quantidade de líquido, o que requer maior atenção quanto ao estado deste paciente. Atentar que a presença de líquido em uma localização não indica que a lesão seja neste lado. É comum a presença de líquido em volume significativo na goteira direita e a lesão originária do líquido ser esplênica (Figura 6).

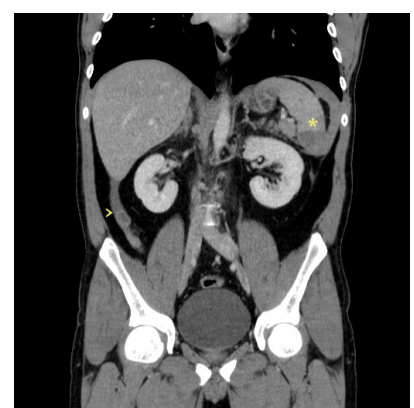

Figura 6. Tomografia computadorizada: presença de líquido na goteira parietocólica direita (>) e a lesão primária esplênica, à esquerda $\left(^{*}\right)$ 
Embora não seja o objetivo principal e conforme acima mencionado, a detecção de lesão parenquimatosa pode ser realizada através da ultrassonografia e há posterior correlação com o achado tomográfico, embora muitas vezes seja subestimado pela ultrassonografia (Figura 7).
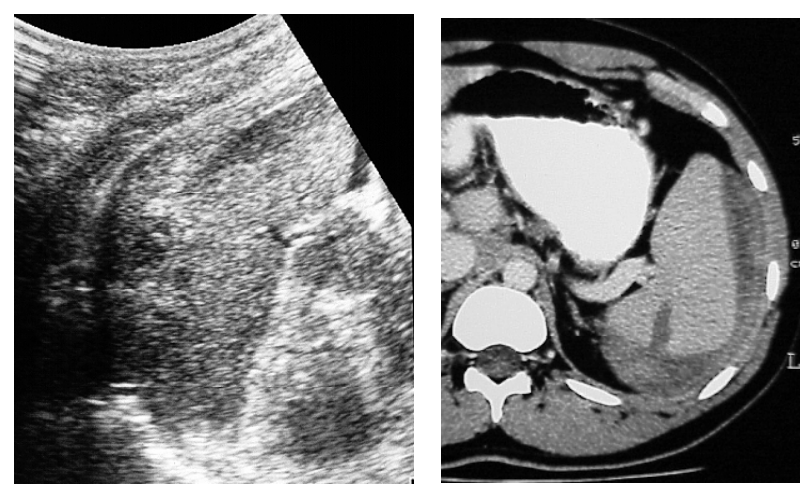

Figura 7. a) Ultrassonografia FAST detectando lesão esplênica com laceração e hematoma periesplênico, b) confirmada pela TC

O protocolo de TC deve sempre ser realizado com a administração de contraste endovenoso. A única contraindicação absoluta é o histórico de reação alérgica grave pregressa. $O$ contraste oral não é realizado, salvo na maior estabilidade do paciente ou quando há o objetivo específico de se pesquisar perfuração em víscera oca. O exame deve ser realizado preferencialmente no equipamento helicoidal e se possível de múltiplos detectores. A vantagem desse equipamento é a aquisição rápida de imagens, de mais de um segmento do corpo e a possibilidade da realização de estudos angiotomográficos, nos quais a obtenção de imagens durante a injeção venosa do meio de contraste permite nítida reconstrução vascular (Figuras 8, 9 e 10).

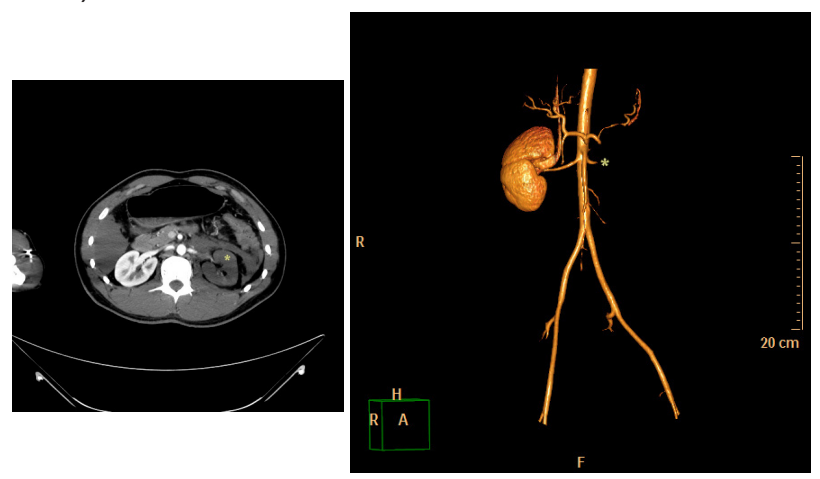

Figura 8. a)Tomografia computadorizada de múltiplos detectores: lesão arterial renal esquerda com hipocontrastação do rim esquerdo (*), b) Reconstrução VR ("volume rendering") da fase arterial demonstrando o local da lesão da artéria renal esquerda $\left({ }^{*}\right)$

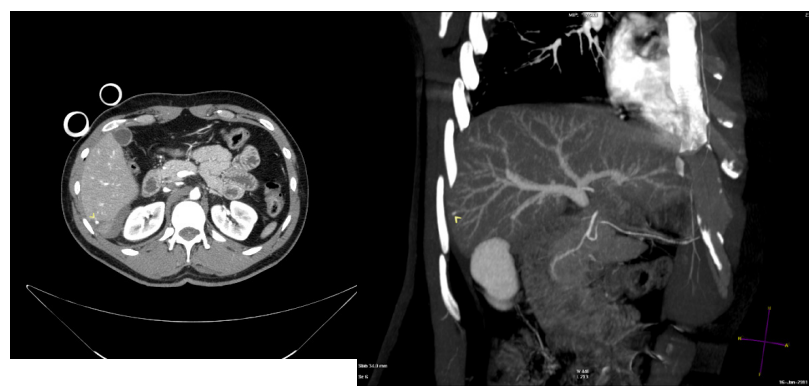

Figura 9. a) Tomografia computadorizada de múltiplos detectores: pequena imagem hipervascularizada no segmento hepático VI (>), b) Reconstrução MIP ("maximal intensity projection") da fase arterial demonstrando pequena lesão arterial ativa $(<)$
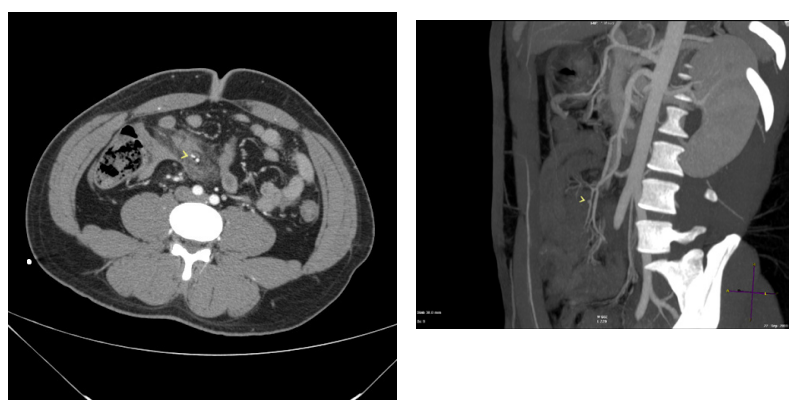

Figura 10. a)Tomografia computadorizada de múltiplos detectores: pequena imagem hipervascularizada junto ao ramo da artéria mesentérica superior ( $>$ ), b) Reconstrução MIP ("maximal intensity projection") da fase arterial confirma o achado como pequena lesão arterial (>)

São realizadas aquisições nas fases arterial, portal e excretora, além da fase pré-contraste, que pode ser opcional, embora sempre recomendada.

A pesquisa ultrassonográfica limita-se à detecção de líquido livre e na eventual detecção de lesão de víscera parenquimatosa, a orientação é prosseguimento no estudo, pois o que se tornou evidente pode ser apenas parte do processo. Portanto, na presença de líquido ou lesão parenquimatosa a investigação tem que ser aprofundada, no caso de instabilidade por hipotensão, a cirurgia deve ser indicada imediatamente.

A TC muda um pouco o modelo tradicional da investigação do trauma abdominal fechado uma vez que a pesquisa agora é ativamente pela lesão, não pela sua consequência (hemoperitônio). Assim, é possível o estadiamento da lesão de acordo com o órgão e a decisão de conduta conservadora mesmo na vigência de lesão intra-abdominal pode ser realizada, inimaginável no contexto anterior (da pesquisa de hemoperitônio).

Neste método o que se procura são alteração da atenuação e descontinuidades nos órgãos parenquimatosos, com variável grau de lesão, caracterizando-se contusões ou lacerações; quando extensas e atravessam o órgão resultam em fratura 
e quando múltiplas com desorganização arquitetural, caracteriza-se explosão. A avaliação também envolve a pesquisa da anatomia vascular e a pesquisa indireta de lesões pela caracterização de hematomas adjacentes aos órgãos afetados. Estudos dirigidos como a cistotomografia podem ser realizados no intuito específico de se achar lesão vesical (Figura 11).

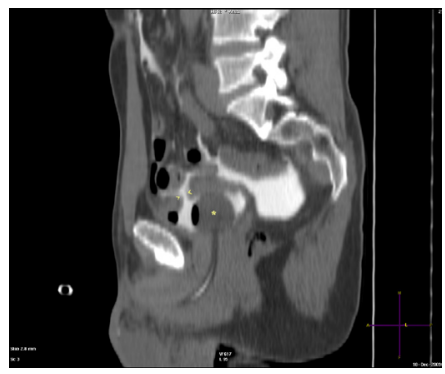

Figura 11. Cistotomografia computadorizada: solução de continuidade no domo vesical (indicada pelas setas), com extravasamento do meio de contraste injetado através da sonda vesical $\left({ }^{*}\right)$

O fígado é o órgão mais acometido nos casos de trauma abdominal. As lesões mais frequentes são as lacerações e os hematomas. À TC as lacerações apresentam-se como soluções de continuidade na superfície do órgão, linhas hipoatenuantes desde a superfície hepática. Já os hematomas são acúmulos sanguíneos que podem ser subcapsulares ou intraparenquimatosos (Figura 9), caracterizando-se como imagens hiperatenuantes junto ao contorno no primeiro caso ou hipo ou hiperatenuantes no segundo caso. A classificação da lesão hepática depende da extensão da laceração e no caso de hematoma, sua localização e dimensão.

$O$ baço é o segundo órgão mais acometido nos casos de trauma abdominal. A classificação das lesões segue próximo à do fígado, caracterizando-se em lacerações e hematomas (Figura 7).

O rim é o terceiro órgão mais acometido nos casos de trauma abdominal. Aqui, além do hematoma perirrenal e das lacerações, a extensão para o sistema coletor também passa a ter importância, bem como a lesão arterial é relevante (Figura 8).

Outras estruturas também merecem atenção especial, destacando-se aqui o pâncreas e o mesentério. No primeiro a atenção é focada na pesquisa de lesões, principalmente se elas envolvem o ducto pancreático, evitando complicações durante a internação; no segundo caso, é o fator envolvido com sangramento ou líquido livre na falta de outra lesão evidente em víscera parenquimatosa.

Os dados obtidos pelos exames passam a subsidiar diretamente a equipe cirúrgica, permitindo a tomada de decisões como se a cirurgia é iminente ou se é possível a conduta clínica, uma vez que a tendência é restringir as cirurgias aos casos necessários, evitando-se laparotomias nãoterapêuticas, muito mais frequentes na época da lavagem peritoneal diagnóstica.

Desta forma, o diagnóstico por imagem exerce importante papel no contexto de trauma abdominal fechado, ressalvando-se que se encaixa perfeitamente no modelo mais amplo de atendimento ao politraumatizado. O protocolo e a recomendação do seu uso foram resumidos na Figura 12.

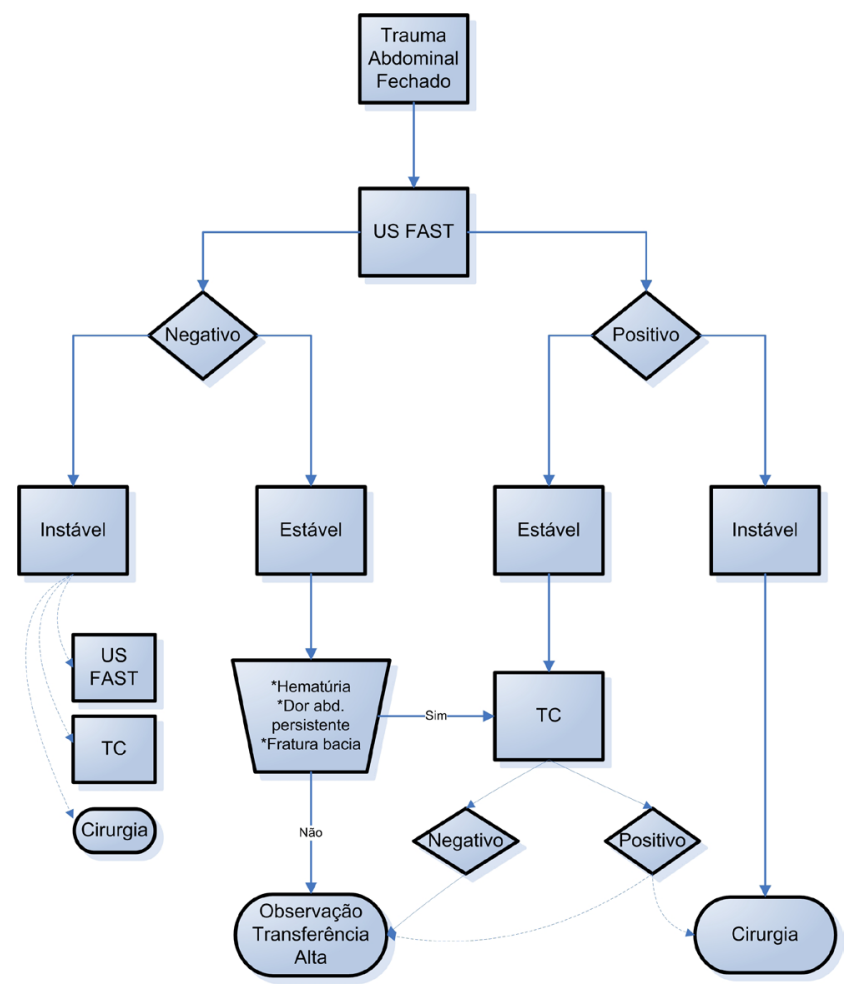

Figura 12. Protocolo de US e TC no atendimento ao trauma abdominal fechado

\section{REFERÊNCIAS}

Demetriades D, Velmahos G. Technology-driven triage of abdominal trauma: the emerging era of nonoperative management. Annu Rev Med. 2003;54:1-15.

Jeffrey RB. Trauma: section 3 abdomen/pelvis. In: Diagnostic imaging - emergency. Salt Lake City: Amirsys; 2007.

Knudson MM, Maull KI. Nonoperative management of solid organ injuries: past, present, and future. Surg Clin North Am. 1999;79:1357-71.

Marincek B, Dondelinger RF, editors. Emergency radiology - imaging and Intervention. Berlin: Springer-Verlag; 2007.

Novelline RA, Rhea JT, Bell T. Helical CT of abdominal trauma. Radiol Clin North Am. 1999;37:591-612.

Rhea JT. CT of abdominal trauma: part I RSNA categorical course. In: Diagnostic radiology: emergency radiology. Oak Brook, III.: RSNA; 2004. p.91-100.

Shanmuganathan, K. CT of Abdominal Trauma: Part I RSNA Categorical course. In: Diagnostic radiology: emergency radiology. Oak Brook, III.: RSNA; 2004. p.101-12. 